\title{
All Maximum Size Two-Part Sperner Systems: In Short
}

\author{
HAROUT AYDINIAN ${ }^{1}$ and PÉTER L. ERDÖS $\mathrm{S}^{2 \dagger}$ \\ ${ }^{1}$ Department of Mathematics, University of Bielefeld, PO Box 100131, D-33501, Bielefeld, Germany \\ (e-mail: ayd@uni-bielefeld.de) \\ ${ }^{2}$ A. Rényi Institute of Mathematics, Hungarian Academy of Sciences, \\ Budapest, PO Box 127, H-1364 Hungary \\ (e-mail: elp@renyi.hu)
}

Received 28 July 2005; revised 18 December 2005

In this note we give a very short proof for the description of all maximum size two-part Sperner systems.

Katona [6] and Kleitman [8] independently observed that the statement of the Sperner theorem remains unchanged if the conditions are relaxed in the following way. Let $X=X_{1} \cup X_{2}$ be a partition of the underlying set $X,\left|X_{i}\right|=n_{i}, n_{1}+n_{2}=n$ (with $n_{1} \geqslant n_{2}$ ). We say that $\mathscr{F}$ is a two-part Sperner family if $E, F \in \mathscr{F}, E \varsubsetneqq F \Rightarrow \forall i:(F \backslash E) \not \subset X_{i}$. It was proved that the size of a two-part Sperner family cannot exceed $\left(\begin{array}{c}n \\ \lfloor n / 2\rfloor\end{array}\right)$. It took 20 years to find all maximum size two-part Sperner families [3]. The description requires some more definitions.

The two-dimensional profile matrix $M(\mathscr{F})$ is defined by $M_{i j}(\mathscr{F})=\#\left\{F \in \mathscr{F}: \mid X_{1} \cap\right.$ $\left.F|=i,| X_{2} \cap F \mid=j\right\}$. This can be considered as a point in the real space $\mathbb{R}^{\left(n_{1}+1\right)\left(n_{2}+1\right)}$. The profile matrices of the two-part Sperner families determine a point set in this space, and it is known that the vertices of their polytope are the profile matrices of the homogeneous systems (see [2, Theorem 3.2]), where a family $\mathscr{F}$ is called homogeneous (with respect to the partition $\left.X_{1}, X_{2}\right)$ if $F \in \mathscr{F}$ implies $E \in \mathscr{F}$ for all sets $E$ satisfying $\forall i:\left|E \cap X_{i}\right|=\left|F \cap X_{i}\right|$. A homogeneous family can be described by the set $I(\mathscr{F})=\left\{\left(i_{1}, i_{2}\right): \forall j:\left|F \cap X_{j}\right|=i_{j}\right.$ for some $F \in \mathscr{F}\}$. If $\mathscr{F}$ is a homogeneous two-part Sperner family, then $I(\mathscr{F})$ cannot contain pairs with the same first or second components, respectively. Consequently we have $|I(\mathscr{F})| \leqslant n_{2}+1$. We say that a homogeneous family $\mathscr{F}$ is full if $|I(\mathscr{F})|=n_{2}+1$. Then, for

$\dagger$ Supported, in part, by the Hungarian NSF, under contract nos. T37846, AT048826.

$\ddagger$ This work was carried out when the second author visited the University of Bielefeld under the Marie Curie FIST Mobility Program. 
every $i_{2}\left(0 \leqslant i_{2} \leqslant n_{2}\right)$, there is a unique $f\left(i_{2}\right)$ such that $\left(f\left(i_{2}\right), i_{2}\right) \in I(\mathscr{F})$. A homogeneous family is called well-paired if it is full, $\left\{f(i): i=0,1, \ldots, n_{2}\right\}$ is the $\left(n_{2}+1\right)$-element interval around $\left\lfloor n_{1} / 2\right\rfloor$ and

$$
\left(\begin{array}{c}
n_{2} \\
i
\end{array}\right)<\left(\begin{array}{c}
n_{2} \\
j
\end{array}\right) \text { implies }\left(\begin{array}{c}
n_{1} \\
f(i)
\end{array}\right) \leqslant\left(\begin{array}{c}
n_{1} \\
f(j)
\end{array}\right)
$$

for every pair $1 \leqslant i, j \leqslant n_{2}$. It is clear that well-pairing is not unique.

Theorem 1 ([3]). Let $\mathscr{F}$ be a two-part Sperner family with parts $X_{1}, X_{2}$. Then

$$
|\mathscr{F}| \leqslant\left(\begin{array}{c}
n \\
\left\lfloor\frac{n}{2}\right\rfloor
\end{array}\right)
$$

with equality if and only if $\mathscr{F}$ is a homogeneous well-paired family.

In [9] and [4] further proofs were given but none were really short or easy to understand. Here we give a very short proof of Theorem 1 .

Proof. Let $F \subset X_{2}$. Then define $\mathscr{F}(F):=\left\{E \subset X_{1}: E \cup F \in \mathscr{F}\right\}$. Now $\mathscr{F}(F)$ is a Sperner family, therefore, due to the well-known LYM (Lubell-Yamamoto-Meshalkin) inequality,

$$
\sum_{E \in \mathscr{F}(F)} \frac{1}{\left(\begin{array}{l}
n_{1} \\
|E|
\end{array}\right)} \leqslant 1
$$

with equality if and only if $\mathscr{F}(F)$ consists of a full level in the subset-lattice of $X_{1}$. (For details see, for example, [7].) Summing this inequality over all $i$-element subsets of $X_{2}$, we have, for all $i=0, \ldots, n_{2}$,

$$
\sum_{F \in\left(\begin{array}{c}
X_{2} \\
i
\end{array}\right)} \sum_{E \in \mathscr{F}(F)} \frac{1}{\left(\begin{array}{c}
n_{1} \\
|E|
\end{array}\right)} \frac{1}{\left(\begin{array}{c}
n_{2} \\
i
\end{array}\right)} \leqslant 1
$$

Finally

$$
\sum_{i=0}^{n_{2}} \sum_{F \in\left(\begin{array}{c}
x_{2} \\
i
\end{array}\right)} \sum_{E \in \mathscr{F}(F)} \frac{1}{\left(\begin{array}{c}
n_{1} \\
|E|
\end{array}\right)\left(\begin{array}{c}
n_{2} \\
i
\end{array}\right)}=\sum_{j, i} \frac{M_{j i}(\mathscr{F})}{\left(\begin{array}{c}
n_{1} \\
j
\end{array}\right)\left(\begin{array}{c}
n_{2} \\
i
\end{array}\right)} \leqslant n_{2}+1 .
$$

The middle term of inequality (4) is a linear function of the profile matrix, and it is easy to check that for all full homogeneous families (including well-paired homogeneous families) inequality (4) holds with equality. Therefore all maximum size two-part Sperner families must also satisfy it with equality.

Indeed, the cardinality of a family is the sum of the entries in its profile matrix. This is a positive linear function of the profile matrices, and therefore its maximum is attained only by points which are on the facets of the polytope spanned by the profile matrices of maximum size homogeneous two-part Sperner families. So the profile matrix of any maximum size two-part Sperner family is a convex linear combination of profile matrices of maximum size homogeneous families, and hence it satisfies any linear equality which is also satisfied by these vertices.

Consequently, for maximum size families inequalities (2) and (3) must hold with equality. Therefore, due to (3), each $F \subset X_{2}$ must be a trace on $X_{2}$ of some element of $\mathscr{F}$. Furthermore, for all $F \subset X_{2}$ the family $\mathscr{F}(F)$ must be a full level in the subset-lattice of $X_{1}$. 
We consider first the case $n_{1}=n_{2}$. In this case we can repeat the reasoning for all $E \subset X_{1}$, which proves that in this case each maximum size two-part Sperner system is homogeneous.

In the case of $n_{1}>n_{2}$, denote by $\mathscr{F}(j) \subset 2^{X_{2}}$ the set of all $F \subset X_{2}$ such that $E \in \mathscr{F}(F)$ with a fixed $E \subset X_{1},|E|=j$. (By the previous statement all $j$-element subsets of $X_{1}$ would define the same set.) Therefore, for all $j \in\left\{1, \ldots, n_{1}\right\}, \mathscr{F}(j)$ is a Sperner family, and $\mathscr{F}(j) \cap \mathscr{F}\left(j^{\prime}\right)=\emptyset$ holds for all $j \neq j^{\prime}$. Furthermore, any $t$ families among them form a $t$-Sperner family (their union does not contain a $(t+1)$-chain), and therefore, by Paul Erdős's theorem [1],

$$
\sum_{k=1}^{t}\left|\mathscr{F}\left(j_{k}\right)\right|
$$

is at most the sum of the $t$ largest binomial coefficients. Now, the cardinality of our maximum size two-part Sperner system is

$$
|\widetilde{F}|=\sum_{j=0}^{n_{1}}\left(\begin{array}{c}
n_{1} \\
j
\end{array}\right)|\mathscr{F}(j)| .
$$

We get the largest value $\left(\begin{array}{c}n \\ \lfloor n / 2\lfloor\end{array}\right)$ in (5) with the following greedy algorithm. Let $B_{0} \geqslant B_{1} \geqslant$ $\cdots \geqslant B_{n_{1}}$ and $A_{0} \geqslant \cdots \geqslant A_{n_{2}}$ be enumerations of the binomial coefficients $\left(\begin{array}{c}n_{1} \\ j\end{array}\right)$ and $\left(\begin{array}{c}n_{2} \\ i\end{array}\right)$, respectively. If $A_{0}>A_{1}$ then we have to match $B_{0}$ with $A_{0}$. We have already matched the pairs up to $k-1$. Now $A_{k}=A_{k+1}$. Then $B_{k}$ and $B_{k+1}$ must be matched with these two items. Recall that the corresponding $\mathscr{F}(j)$ and $\mathscr{F}\left(j^{\prime}\right)$ families are Sperner families, and so, by the LYM inequality, they may have that many elements if and only if each of the two families is equal to one full level in the subset lattice of $X_{2}$. So we get that every maximum size two-part Sperner family must be well-paired.

The authors are indebted to the unknown referee for his help.

\section{References}

[1] Erdős, Paul (1945) On a lemma of Littlewood and Offord. Bull. Amer. Math. Soc. 51 898-902.

[2] Erdős, P. L. and Katona, G. O. H. (1986) Convex hulls of more-part Sperner families. Graphs Combin. 2 123-134.

[3] Erdős, P. L. and Katona, G. O. H. (1986) All maximum 2-part Sperner families. J. Combin. Theory Ser. A 43 58-69.

[4] Erdős, P. L., Füredi, Z. and Katona, G. O. H. (2005) Two-part and $k$-Sperner families: New proofs using permutations. SIAM J. Discrete Math. 19 489-500.

[5] Füredi, Z., Griggs, J. R., Odlyzko, A. M. and Shearer, J. M. (1987) Ramsey-Sperner theory. Discrete Math. 63 143-152.

[6] Katona, G. O. H. (1966) On a conjecture of Erdős and a stronger form of Sperner's theorem. Studia Sci. Math. Hungar. 1 59-63.

[7] Katona, G. O. H. (2000) The cycle method and its limits. In Numbers, Information and Complexity (Althöfer et al., eds), Kluwer, pp. 129-141.

[8] Kleitman, D. J. (1965) On a lemma of Littlewood and Offord on the distribution of certain sums. Math. Z. 90 251-259.

[9] Shahriari, S. (1996) On the structure of maximum 2-part Sperner families. Discrete Math. 162 229-238. 